\title{
Magnetic Stripes on Hexagonal Lattice with Competing Exchange and Dipole-Dipole Interactions
}

\author{
A. Joknys And E.E. Tornau \\ Semiconductor Physics Institute \\ Goštauto 11, LT-01108, Vilnius, Lithuania
}

\begin{abstract}
We obtained the phase diagram for the Ising model with competing exchange and dipolar interactions on 2D hexagonal lattice. By using histogram method we determined the order of the phase transition from isotropic stripe phase to low-temperature anisotropic stripe phases AF $h$ of different stripe widths $h$. The first order phase transition was found to AF1 and AF2 phases and the second order - to AF3 and AF4 phases. We have also found that in AF1 phase stripe domain grows with time as $t^{0.5}$. In phases AF3 and AF4 stripe domain growth is proportional to $\log t$.
\end{abstract}

PACS numbers: 61.46.Bc, 68.18.Jk, 68.35.Rh

\section{Introduction}

In ultrathin magnetic films and nanostructures long-range dipolar interactions between magnetic spins are as important as short-range exchange interactions. When these two forces are competing, the periodic striped structure with alternating antiferromagnetic domains might occur in the system. If dipole interactions dominate, the stripes are thin, but when short-range interactions are prevailing the stripes are thick or the ferromagnetic (FM) phase occurs. The best known experimental realization of a magnetic striped system with magnetization perpendicular to film plane is ultrathin films of $\mathrm{Fe} / \mathrm{Cu}(001)$ [1]. When surface anisotropy of ultrathin film prevails over in-plane anisotropy, its magnetic moments have one-to-one correspondence to the Ising spins. This fact allows for a simple theoretical description of magnetic properties of these films in terms of the Ising model with dipolar term decaying with distance as $r^{-3}$. The phase transition from long-range order stripe phase to isotropic short-range-order stripe phase was first studied on a square lattice $[2,3]$. The only study of this model on a hexagonal lattice was performed in Ref. [4]. The authors tried to demonstrate the KosterlitzThouless mechanism of transition at the stripe melting point. However, neither 
the transition order nor the dynamics of stripe domain relaxation were studied for this model on a hexagonal lattice. Analogy of this model and planar model with symmetry breaking orientational field allows to suggest [5] that stripe melting transition on a square and hexagonal lattices might be quite different.

\section{Model and phase diagram}

In this paper we study the Ising model with competing exchange and dipolar interactions on a hexagonal lattice. The Hamiltonian has the form

$$
\mathcal{H}=-J \sum_{\langle i \neq j\rangle} s_{i} s_{j}+D \sum_{\langle i \neq j\rangle} \frac{s_{i} s_{j}}{r_{i j}^{3}},
$$

where $J$ is a FM interaction constant and the first sum is restricted to pairs of the nearest neighbor spins. In the second sum $D$ is dipolar interaction constant and site indices $i$ and $j$ run over all $N$ sites of a hexagonal lattice. In Hamiltonian (1) we consider the sum over every pair of spins in dipolar term just once. The spin variables $s_{i}= \pm 1$ are supposed to be aligned out of plane. Further, everywhere the temperature $T=k_{\mathrm{B}} T($ in $\mathrm{K}) / D$ and the ratio of interaction parameters $\eta=J / D$ are in dimensionless units.

To obtain the phase transition (stripe melting) point $T_{\mathrm{c}}$ we calculate the temperature dependence of the energy per spin $E=\mathcal{H} / N$ and the specific heat $C_{V}=\left(1 / T^{2}\right)\left(\left\langle E^{2}\right\rangle-\langle E\rangle^{2}\right)$ of the model (1) for four main phases AF $h$ with stripe width $h=1,2,3$ and 4 (in $a$ units). We perform the calculations by Monte Carlo method using Metropolis algorithm on a hexagonal lattice of $N=48 \times 48$ spins, including the long-range dipolar interactions up to the distance of $24 a$.

The energy in the phase transition region of phases AF1 and AF2 changes abruptly, in contrast to the $E(T)$ dependence for thicker stripe phases which is much stronger influenced by the fluctuations. Correspondingly, the peaks at $T_{\mathrm{c}}$ of $C_{V}(T)$ are sharp and narrow at the transitions to AF1 and AF2 phases and more flat at those to AF3 and AF4 and even thicker stripe phases (Fig. 1). The same tendency might be seen in temperature dependences of order parameter and susceptibility which are also good indications of the $T_{\mathrm{c}}$ point. Using thus obtained $T_{\mathrm{c}}$ data at different $\eta$ values we also present in Fig. 1 the phase diagram of the model (1) up to AF5 phase. Determination of the stripe phases with $h>4$ for hexagonal lattice is rather difficult, because thicker stripes do not anymore maintain the stripe configuration with fixed thickness (see insets for Fig. 1).

\section{Transition order and dynamics}

Analysis of temperature dependences of main transition parameters indicates that in AF1 and AF2 phases the stripe melting transition is, most likely, of the first, while in the $h>2$ phases - of the second order. To determine the order of transition in Monte Carlo calculations more accurately, we had to employ energy histogram method [6] and calculate the energy distribution $P(E)$ at the phase transition point. We obtained [7] twin-peak $P(E)$ structure at the $T_{\mathrm{c}}$ point to 

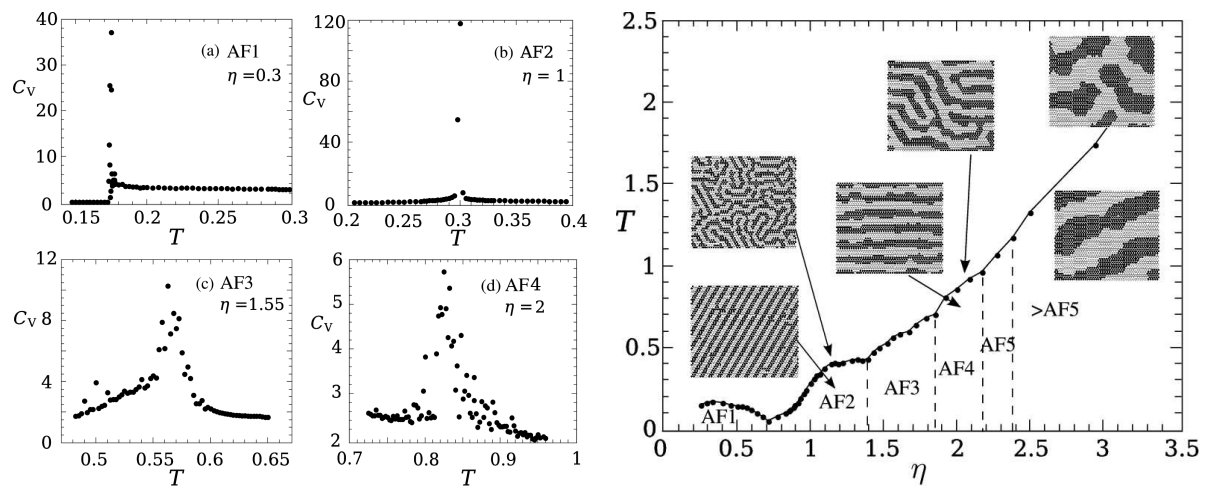

Fig. 1. (left, (a)-(d)) Temperature dependences of specific heat close to the $T_{\mathrm{c}}$ point to four main stripe phases. (right) Phase diagram. Snapshots of stripe domains just above and below phase transition points for $\eta=1.1$ (AF2), $\eta=2$ (AF4) and $\eta=3.3$ (AF8-12) are given as insets.

the AF1 and AF2 phases and single peak structure to the AF3 and AF4 phases. Therefore, the transitions to the AF1 and AF2 phases should be attributed to the first order, and the transitions to thicker stripe phases - to the second order phase transitions. The tricritical point separating first and second order transitions on the phase diagram was found to be roughly at the AF2 and AF3 phase boundary.

We have also studied the dynamical properties of the model (1) for phases $\mathrm{AF} 1, \mathrm{AF} 2, \mathrm{AF} 3$, and AF4. The system was annealed at high temperatures and then quenched to temperatures below $T_{\mathrm{c}}$. We monitored how the energy $E(t)$ of a disordered system relaxes to the ground state stripe phase energy $E(\infty)$, here $t$ is the time measured in Monte Carlo steps per site $(\mathrm{MCS} / \mathrm{s})$. The final curves were obtained by averaging over 20-50 $E(t)$ dependences. For the Isingtype models the size of a FM domain $R$ grows with time as $R \sim \Delta E_{N}^{-1} \sim t^{0.5}$, where $\Delta E_{N}=[E(t)-E(\infty)] /[E(0)-E(\infty)]$. In Figs. 2a and b we show $\Delta E_{N}(t)$ dependence for three values of the interaction parameter $\eta(0.25,0.33$, and 0.5$)$ which are in the AF1 phase region of the phase diagram. For any chosen temperature inside the AF1 phase, the data points for all three values of $\eta$ fall onto one curve, indicating that the dynamics inside the AF1 phase is the same for all $\eta$ values, and the domain growth in this phase follows the $\sim t^{0.5}$ law. The dynamics in the AF2 phase indicates some saturation for higher quench temperature as in the AF1 phase, but the relaxation is clearly slower [7]. We consider this behavior as intermediate between the Ising-type of dynamics of the AF1 phase and much slower dynamics of the thicker stripe phases presented in Fig. 2c. The dynamics of AF3 and AF4 phases is slow and up to $10^{5} \mathrm{MCS} / \mathrm{s}$ clearly follows the $\Delta E_{N}(t) \sim(\log t)^{-1}$ dependence. This law still holds for AF5 and AF6 phases. 


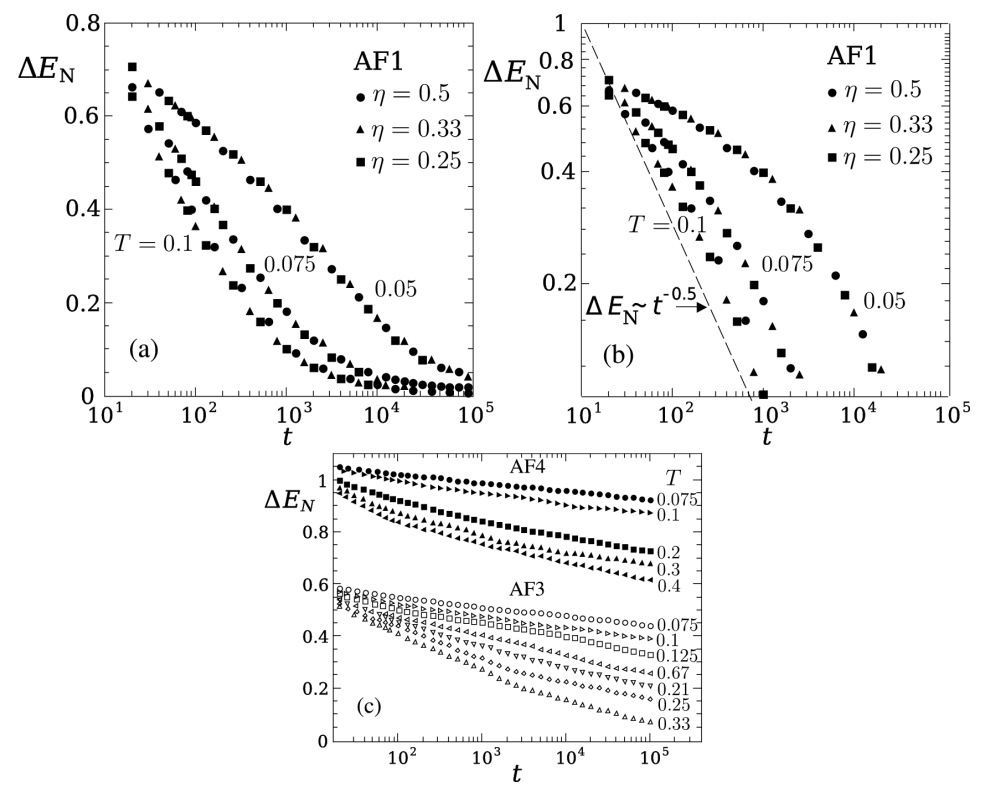

Fig. 2. Energy relaxation for three $\eta$ values in the AF1 phase and three values of quench temperature: semilog plot (a) and $\log -\log$ plot (b). Dashed line shows the Ising model dynamics. (c) Semilog plots of energy relaxation in the AF3 $(\eta=1.67)$ and AF4 $(\eta=2)$ phases. AF4 curves are vertically shifted for clarity by 0.5 .

\section{Acknowledgments}

This work was partly supported by project MULTIMA (No. C-18/2007) of Lithuanian State Science and Studies Foundation.

\section{References}

[1] R. Allenspach, A. Bischof, Phys. Rev. Lett. 69, 3385 (1992); A. Vaterlaus, C. Stamm, U. Maier, M.G. Pini, P. Politi, D. Pescia, Phys. Rev. Lett. 84, 2247 (2000)

[2] A.B. MacIsaak, J.P. Whitehead, M.C. Robinson, K. De' Bell, Phys. Rev. B 51, 16033 (1995).

[3] I. Booth, A.B. MacIsaac, J.P. Whitehead, K. De'Bell, Phys. Rev. Lett. 75, 950 (1995).

[4] A.D. Stoycheva, S.J. Singer, Phys. Rev. Lett. 84, 4657 (2000).

[5] J.V. Jose, L.P. Kadanoff, S. Kirkpatrick, D.R. Nelson, Phys. Rev. B 16, 1217 (1977).

[6] J. Lee, J.M. Kosterlitz, Phys. Rev. Lett. 65, 137 (1990).

[7] A. Joknys, E.E. Tornau, Lithuanian J. Phys. 47, 321 (2007). 